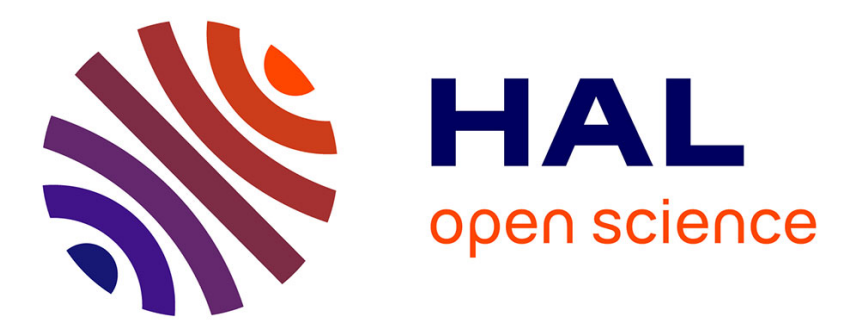

\title{
Une stèle de Montceau-les-Mines montrant un chat domestique
}

\author{
Louis Armand-Calliat
}

\section{To cite this version:}

Louis Armand-Calliat. Une stèle de Montceau-les-Mines montrant un chat domestique. Gallia - Fouilles et monuments archéologiques en France métropolitaine, 1953, 11 (1), pp.85-89. 10.3406/galia.1953.1323 . hal-01921506

\section{HAL Id: hal-01921506 https://hal.science/hal-01921506}

Submitted on 3 Mar 2020

HAL is a multi-disciplinary open access archive for the deposit and dissemination of scientific research documents, whether they are published or not. The documents may come from teaching and research institutions in France or abroad, or from public or private research centers.
L'archive ouverte pluridisciplinaire HAL, est destinée au dépôt et à la diffusion de documents scientifiques de niveau recherche, publiés ou non, émanant des établissements d'enseignement et de recherche français ou étrangers, des laboratoires publics ou privés.

\section{(이) $\$$}

Distributed under a Creative Commons Attribution - NonCommercial - NoDerivatives| 4.0 
parfaitement exécuté me parait accuser l'époque mérovingienne. Ce fragment appartenait au rebord circulaire d'une cuve qui pourrait avoir environ $0^{\mathrm{m}}, 80$ à $1^{\mathrm{m}}$ de diamètre, une cuve baptismale peutêtre.

Après le mur sud, le mur nord subit la même opération de démolition. Il ne donna rien de nouveau jusqu'au niveau du sol, mais, en arrivant aux fondations, les ouvriers trouvèrent, au milieu de la vieille maçonnerie qui tombait en poussière, deux massifs compacts que leur pioche n'entamait pas. Et tout naturellement émergèrent ainsi deux murs épais, recouverts d'un magnifique béton rose (ép.: $0^{\mathrm{m}}, 30$ ), auquel adhéraient encore des plaques de parement de fine pierre blanche. Peu à peu, entre ces deux murs, se dessina le fond d'une construction en forme d'octogone légèrement aplati, mesurant environ $2^{\mathrm{m}}, 50$ de long sur $2^{\mathrm{m}}$ de large. Sur une des faces, 3 marches de descente subsistent encore. Le fond de cette cuve octogonale sonnait le creux. Par un trou pratiqué dans le béton, j'aperçus les colonnes alignées d'un hypocauste intact. Il s'étend sur la cuve octogonale qu'il déborde même un peu: Les pilettes en briques sont intactes, les plus près de l'entrée du four presque entièrement rongées par le feu. Le fond et le plafond étaient tapissés de suie. Ia gueule du four est tournée vers le Nord. Sur le pourtour, les buses de prise d'air chaud pour le chauffage des murs sont toutes en place. Un tuyau d'écoulement d'eau, traversant l'épaisseur des murs et descendant de la pièce octogonale supérieure prouve que celle-ci était une piscine.

J'ai trouvé également de nombreux fragments d'entablements moulurés en marbre blanc, en marbre gris et vert antique. L'examen attentif de l'église me révéla enfin que le transept nord était construit sur des fondations galloromaines que des sondages ont en effet retrouvées et, fait plus remarquable encore, que la base des murs qui soutiennent le clocher n'est autre qu'un pan de mur antique, conservé jusqu'à $2^{\mathrm{m}}, 50$ de hauteur. L'appareil et tous les joints en ciment rose en font foi.

\section{P.-M. TONNEI,IIER.}

\section{Une stèle de MoNTCEAU-LES-Mines MONTRANT UN CHAT DOMESTIQUE}

La région de Montceau-les-Mines, qui passait naguère pour pauvre en sculptures gallo-romaines, nous avait réservé en 1951 la surprise de trois monuments inédits, retrouvés dans une maison précédemment habitée par un archéologue du siècle dernier ${ }^{1}$. Or voici qu'une stèle intéressante vient encore d'être identifiée près de la même ville. Mise au jour en 1937 au hameau de Lucy, sur le bord de la voie romaine reliant, croit-on, Autun à Belleville par Tramayes, elle était restée abandonnée et inaperçue lorsque, l'été dernier, un érudit local, M. Jean Bernardin, la remarqua, la fit mettre à l'abri au musée minéralogique des Houillères et nous en communiqua une photographie pour nous permettre de l'étudier.

C'est un relief (fig. 1) de forme pyramidale, mesurant $0^{\mathrm{m}}, 69$ de hauteur sur $0^{\mathrm{m}}, 46$ de largeur à la base, $0^{\mathrm{m}}, 31$ au sommet et $0 \mathrm{~m}, 266 \mathrm{~d}$ 'épaisseur. Comme le monument funéraire déjà rencontré à Montceau, il est fruste par derrière et beaucoup plus épais en bas qu'en haut, ce qui donne aux deux pièces en question un certain air de parenté, car les stèles de la région chalonnaise ont d'ordinaire à leur revers un aspect différent.

(1) Voir Gallia, IX, 1951, p. 60-63. 


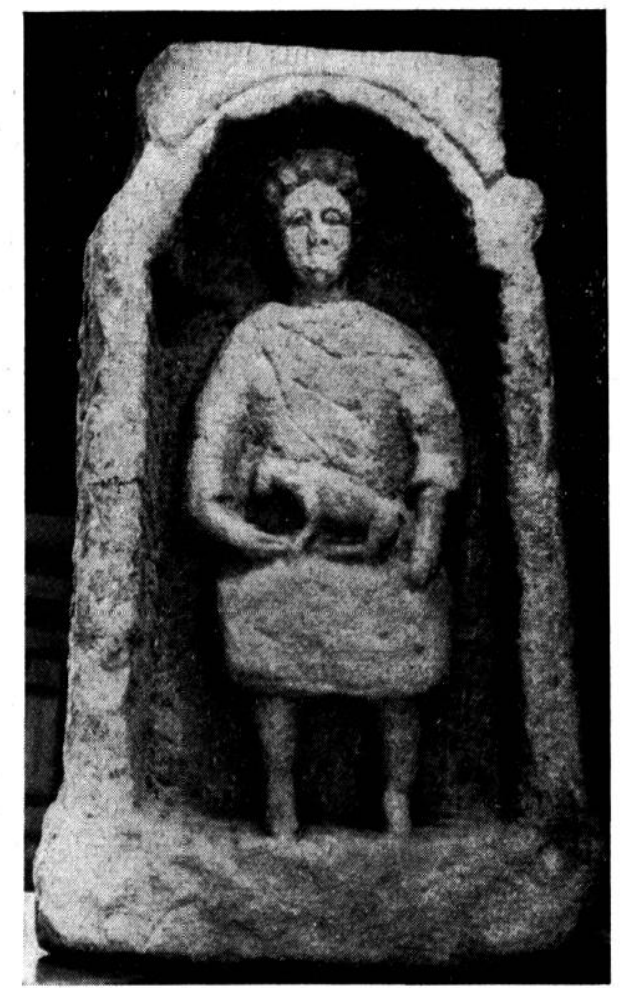

FIr. 1. - Stèle funéraire découverte en 1937 a Montceau-les-Mines (haut.: 0 m. 69).

Dans une niche, arrondie intérieurement en forme de coquille et flanquée à l'extéricur de deux crochets, dont l'un est mutilé, apparaît une fillette assise. vêtue d'une tunique aux plis indiqués sur la poitrine par de simples traits 2 . Le visage est encadré d'une chevelure abondante et boucléc avec une grande régularité. Sur ses genoux, l'enfant tient un quadrupède à longue queue qui ne peut être qu'un chat, comme le montre le profil de la tète (fig. 2).

Originale par sa forme, la stèle de Iontceau, dont la destination funéraire ne saurait guère être mise en doute, nous parait surtout précieuse en raison de la

(2) Cette lechnique se retrouve sur une autre stìle funéraire chalonnaise à Rully (Esp'ERANDLU:-IANTIER, Recueil, XIII, 8.255).

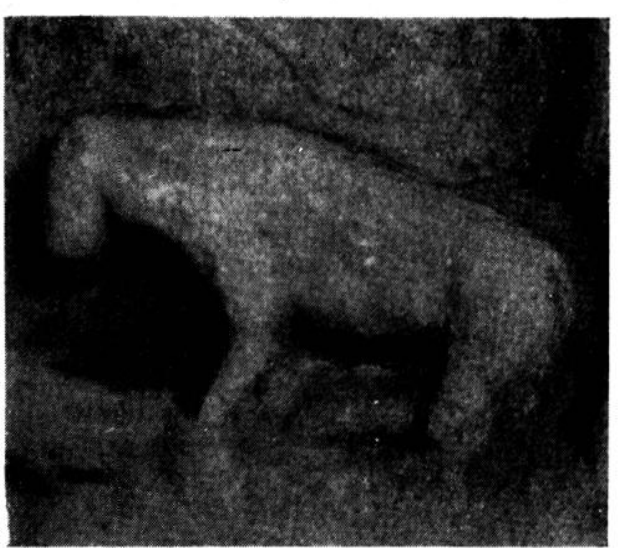

Fsa. 2. - Détail de la stèle montrant le chat domestique.

présence du chat domestique. En effet, parni les milliers de sculptures galloromaines actuellement publiées, il n'en est que cing où l'on reconnaisse cet animal.

Nous mentionnerons en premier lieu trois stèles funéraires d'enfant, découvertes à I,yon, à Dijon et à Bordeaux.

Sur la sculpture de Lyon (EspÉraxdiec, n ${ }^{\circ}$ 1783) qui ressemble de très près à celle de Montceau, on lit une épitaplse nous apprenant que le monument fut érigé à la mémoire d'une enfant de six ans et demi, la petite Aurelia Satyra, par Marullina, sa mère. Nous sommes en présence, par conséquent, d'une simple pierre tombale où la fillette est figurée comme à Montceau avec un jeune chat sur ses genoux (fig. 3). Quant à la stèle de Dijon (E., 3500); elle montre l'enfant debout, tenant d'une main son chat et de l'autre son fouet. Sur le relief de Bordeaux (E., 1193), la fillette presse contre sa poitrine un chat dont un coq, animal familier qui est à terre, becquète la queue (fig. 4).

I.es autres sculptures proprement ditos que l'on peut citer ont une moindre valeur documentaire: ine slatuette de 


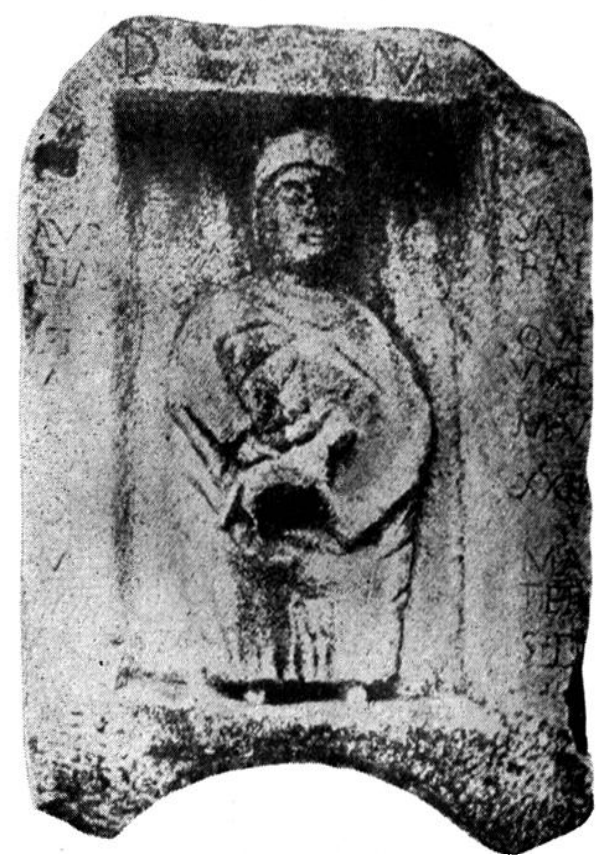

Fli. 3. -- Stèle de Lỹon.

pierre, trouvée à Auxerre (K., 2906), où l'on voit seulement un jeune chat dont le cou est orné d'un large collier; et un haut-relief (E., 7811) découvert à Alise, qui nous donne l'image d'un jeune garfon (Priape ?) debout et nu, tenant un chat pourvu d'une clochetle suspendue à son collier ${ }^{3}$.

Dans le domaine de la petite statuaire, on ne saurait omettre non plus les figurines céramiques de la Gaule romaine ${ }^{4}$, ni les petits bronzes 5 , parmi lesquels

(3) En commentant ce monument devant l'Académie des Inscriptions, Espérandieu remarquait que «le chat n'est indiqué pour aucun dieu », mais que Priape avait des clorhettes pour prévenir de la présence des voleurs» (Comptes rendus de l'Acad. des Inscript., 1937, p. 307-308).

(4) M. Ad. BLANchet, Etude sur les figurines..., dans Mém. des Antiquaires de France, 1891, p. 203, mentionne formellement le chat. En revanche, cet animal est absent de la céramique sigiliée.

(5) S. Rerwach, Rép. de la Statuaire, t. IV, p. 479.

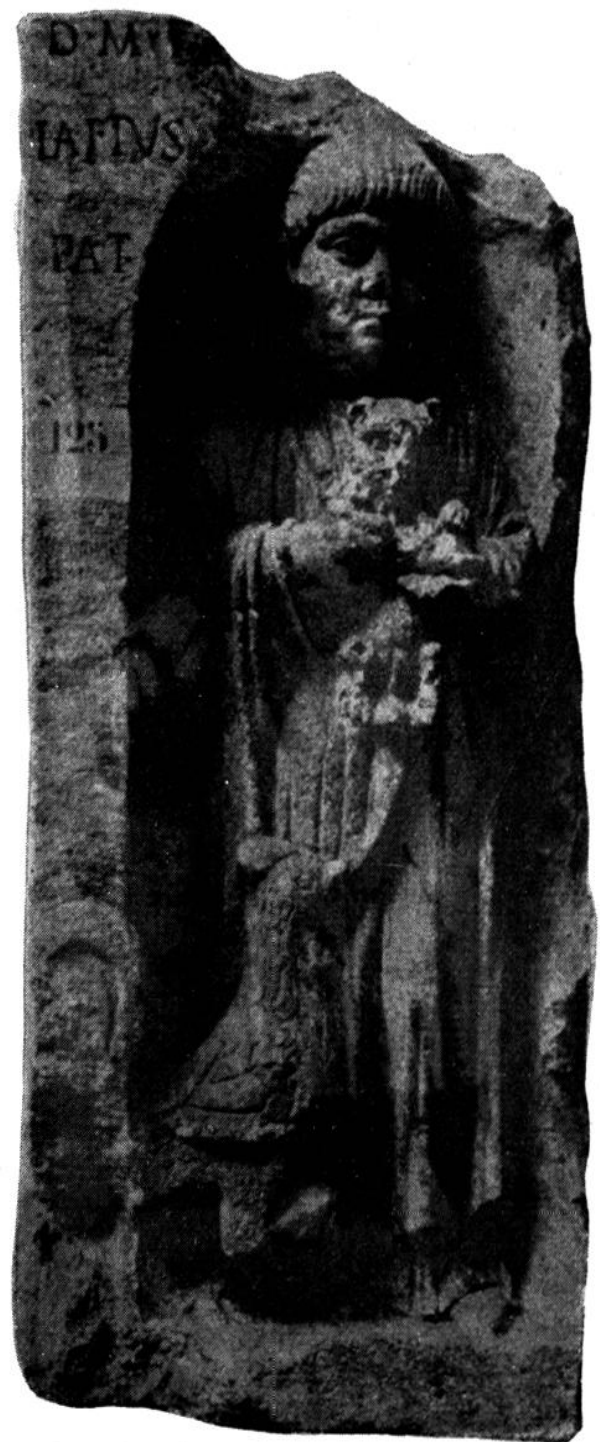

Fis. 4. - Stèle de Bordeaux.

il faut mentionner toul spécialement l'exemplaire si cxpressif, trouvé aux Fins d'Annecy en 1910. Sans doute se peut-il que cette charmante statuette soit importée. Mais il ne saurait en être de même pour les produits en terre blanche dont l'origine est presque toujours gallo-romaine. Tout au plus pourrait-on admettre que l'on est ici en présence.

(6) Ch. Manteacx, Boutae, Annecy, 1913. p. 142 et Rersach, o. l., t. V, p. 421, $n^{\text {ns }} 4-5$. 
d'animaux exotiques montrés en public par des bateleurs, tel ce chat, dont un baš-relief conservé à Rome représente le dressage 7. Dans la même série céramique où l'influence hellénistique est patente, figurent en effet des singes, des tortues, des panthères et des lions, à côté des bêtes de chez nous comme le chien (domestiqué dès le début du Néolithique), le lapin, le taureau, le cheval, le mouton, le bouc et des oiseaux divers.

L'existence du chat domestique en Gaule n'en est pas moins certaine et sa présence sur les quatre monuments funéraires de Lyon, de Montceau, de Dijon et de Bordeaux prouve qu'il était l'un des animaux préférés des enfants d'alors, principalement des petites filles.

On peut remarquer que, sur les six monuments lapidaires de toute nature actuellement connus représentant des chats, cinq ont été mis au jour dans l'Est de la Gaule et le sixième dans un port méridional de l'Ouest, à Bordeaux. Ia Bourgogne et le Lyonnais fournissent la presque totalité des documents. Peut-ître serait-on autorisé à en conclure que le chat domestique, comme on s'y attendait, fut vraisemblablement introduit en Gaule par la vallée du Rhône et de la Saône, plus encore que par les voies maritimes contournant l'Espagne. Il est possible que ce fût sans emprunter forcément le chemin de la péninsule italique où cet animal était très peu fréquent, comme le montrent la rareté des monuments figurés, l'indigence relative des textes et l'examen des ossements découverts au cours des fouilles 8 . Sur le plan linguis-

(7) S. Reinach, Rép. des Reliefs, t. III, p. 213; Saglio-PotTier, Dict. des Antiquités, p. 696, fig. 836, art. de Covgixy et Saglio.

(8) S. ReINACH, Gazette des Beani-Arts, 1900, I, p. 263-264. Cf. ENgelyaxx, Jarbuch des Instituts, 1899, p. 136-137. Covany et $\mathrm{S}_{\text {- }}$ GLio, art. Bestiae masuetae, cicures dans le Dict. des Antiquites, p. 696-699. tique, on observera que le latin classique n'a que le nom du chat sauvage, feles (Ernout-Meillet, I, p. 163). Cattus, qui n'apparaît qu'à la fin du IV ${ }^{\mathrm{e}} \mathrm{s}$ après J.-C. dans Palladius (De re rustica, IV, 9) est probablement celtique. Le gentil compagnon de nos demeures aurait-il été amené directement chez nous d'Egypte, pays qui entretint des relations fréquentes et étroites avec la vallée du Rhône ? Il y a eu dans l'antiquité deux centres de domestication du chat : la Chine (?), et surtout l'Egypte. La Chine ne pouvant être retenue parce que trop lointaine, il ne reste que le pays des pharaons. Or le culte d'Isis, organisé dans plusieur's villes, de Marseille à Lyon ${ }^{9}$ et les figurines ígyptiennes ou égyptisantes 10 retrouvées notamment dans les Bouches-duRhône, le Gard, le Vaucluse, la Drôme, et, plus au Nord, à Lyon, Trévoux, Anse, Belleville, Ia Chapelle-de-Guinchay (bois de Loêze), La Truchère, Ouroux, SaintGermain-du-Plain, Chalon - sur - Saône, Autun, Bourg, Vésines et Conliège, constituent des indices peu contestables, du moins pendant l'occupation latine, de ces rapports entre les rives du Nil et le bassin rhodanien. Venu d'Asie Mineure, le christianisme prendra la même route, la mer et les fleuves navigables étant alors les grandes voies de communication, celles que suivaient le commerce et les

(9) Jullian, Hist. de la Gaule, t. VI, p. 90. (10) E. Guinet, Les Isiaques de la Gaule, dans Rev. arch., $3^{\text {e }}$ série, t. XXXVI, 1900, I, p. 75-86; $4^{\mathrm{e}}$ série, t. XX, 1912, II, p. 197-208; 5e série, t. III, 1916, I, p. 184-210. G. Jeanton, Bull. arch. du Comité des Travaux hist., 1920, p. Clxx. L. ARMand-Calliat, Objets égyptiens ou égyptisants trouvés dans le Chalonnats, dans les Mem. de la Soc. d'Hist. et d'Arch. de Chalon, t. XXIII, 1928-1929, p. 79-86. R. LANTIER, Bull. de la Soc. Nat. des Antiquaires de France, 1942, p. 190. M. H. Rolland, mème Bull., 1948, p. 35, a fait connaitre un sistre égyptien, trouvé à Saint-Rémy-de-Provence et qui serait orné d'une chatte. 
idées 11. Il est assez piquant de constater que, vers l'embouchure de la Garonne, on note à la fois, bien qu'à un moindre degré, des objets égyptiens ou égyptisants, à Royan et à Bazas 12, et la présence du chat sur une stèle funéraire de Bordeaux.

D'après les naturalistes, le chat sauvage de nos pays (felis s!llrestris), animal inapprivoisable par excellence, est entièrement différent du chal domestique (felis domestica). Ce dernier" "n'est arrivé dans nos régions d'une façon importante que vers le $\mathrm{IX}^{\circ}$ siècle ", ont bien voulu nous dire M. Urbain, professeur au Museum, et son collaborateur M. Ed. Dechambre 13. Auparavant, ajouterons-nous, quelques individus semblent avoir suivi vers notre Saône, du II ${ }^{*}$ au Ive siècle, le même itinéraire que le papyrus, les oushabtis et les figures isiaques, sans oublier les idées chrétiennes; et cela clandestinement, car, pour des raisons religieuses, les Egyptiens tenaient tellement a leurs chats qu'ils en prohibaient l'ex-

(11) E. Male, La fin du Paganisme en Gaule, (1950), p. 9-14. Pareillement, c'est en empruntant la vallée du Guadalquivir que le christianisme pénétra en Espagne.

(12) P. Grimal, Gallia, VII, 1, 1949 (1951), p. 131. M. Labrousse, Rev. arch., 6e série, t. XL, 1952, 2, p. 93-95.

(13) Nous devons les remercier ici, ainsi que M. le professeur P. Louis, de la Faculté des Lettres de Lyon, et M. J. de La Comble, conservateur du Musée d'histoire naturelle d'Autun. portation. "C'est seulement après le triomphe du christianisme que les chats purent se répandre en Europe ", estimait Salomon Reinach qui, reprenant une hypothèse de Victor Hehn, liait la diffusion du chat à celle de la doctrine évangélique dans le monde romain ${ }^{14}$. Celte introduction d'un nouvel animal domestique répondait à des besoins nouveaux : pour la chasse aux rongeurs indésirables, on avait employé jusqu'alors des belettes à demi-apprivoisées: elles suffisaient à combattre les souris, les mulots et les campagnols, seuls connus de l'Europe antique vecidentale. L'invasion du rat noir asiatique, sans doute amené par les Barbares, vers le yr ${ }^{\circ}$ ou le $\mathrm{IV}^{\circ}$ siècle, rendit nécessaire l'intervention d'un adversaire plus efficace $\mathbf{1 5}$. On le trouva dans le chat égyptien, libéré précisément par l'affaiblissement ou la disparition des croyances nationales païennes.

La stèle de Montceau-les-Mines conserve l'image de l'un des premiers specimens de cette race féline qui paraît avoir trouvé en Gaule un accueil d'autant plus cmpressé que notre pays fut l'un des plus exposés de l'Empire à la migration du rat, si celle-ci est licee, comme on le croit, aux incursions des Barbares.

\section{Jouis Armand-Calliat.}

(14) S. Reinach, Orpheus, 1909, p. 45, et Gazette des Beaux-Arts, 1900, I, p. 264.

(15) René Thévenis, Origine des animaux domestiques, Paris, 1947, p. 43. 